\title{
Simultaneous, Full Characterization of a Single-Photon State
}

\author{
Tim Thomay, ${ }^{1}$ Sergey V. Polyakov, ${ }^{2}$ Olivier Gazzano, ${ }^{1}$ Elizabeth Goldschmidt, ${ }^{1,3}$ \\ Zachary D. Eldredge, ${ }^{1,4}$ Tobias Huber, ${ }^{1}$ Vivien Loo, ${ }^{1}$ and Glenn S. Solomon ${ }^{1,2, *}$ \\ ${ }^{1}$ Joint Quantum Institute, National Institute of Standards and Technology, \\ \& University of Maryland, Gaithersburg, Maryland 20899, USA \\ ${ }^{2}$ National Institute of Standards and Technology, Gaithersburg, Maryland 20899, USA \\ ${ }^{3}$ US Army Research Laboratory, Adelphi, Maryland 20782, USA \\ ${ }^{4}$ Joint Center for Quantum Information and Computer Science, University of Maryland, \\ \& National Institute of Standards and Technology, College Park, Maryland 20742, USA \\ (Received 25 January 2017; revised manuscript received 8 September 2017; published 15 November 2017) \\ As single-photon sources become more mature and are used more often in quantum information, \\ communications, and measurement applications, their characterization becomes more important. Single- \\ photon-like light is often characterized by its brightness, as well as two quantum properties: the suppression \\ of multiphoton content and the photon indistinguishability. While it is desirable to obtain these quantities \\ from a single measurement, currently two or more measurements are required. Here, we show that using \\ two-photon $(n=2)$ number-resolving detectors, one can completely characterize single-photon-like states \\ in a single measurement, where previously two or more measurements were necessary. We simultaneously \\ determine the brightness, the suppression of multiphoton states, the indistinguishability, and the statistical \\ distribution of Fock states to third order for a quantum light source. We find $n \geq 3$ number-resolving \\ detectors provide no additional advantage in the single-photon characterization. The new method extracts \\ more information per experimental trial than a conventional measurement for all input states and is \\ particularly more efficient for statistical mixtures of photon states. Thus, using this $n=2$, number-resolving \\ detector scheme will provide advantages in a variety of quantum optics measurements and systems. \\ DOI: 10.1103/PhysRevX.7.041036 \\ Subject Areas: Quantum Physics, \\ Quantum Information
}

\section{INTRODUCTION}

Single-photon light is a central element of emerging quantum information systems such as quantum repeaters [1-4] and bosonic logic [5-10]. This nonclassical light is also used in quantum measurement protocols. Such protocols offer advantages over classical measurement protocols for classical properties [11,12], such as in accuracy and sensitivity [13-15], and clearly quantum measurement protocols are essential to access quantum properties.

Light has been traditionally characterized by its coherence properties through a series of normalized Glauber functions $g^{(n)}$, where $2 n$ is the field correlation order [16]. The brightness is given by the unnormalized $g^{(1)}$ function [16], and the normalized second-order correlation function $g^{(2)}$ gives the likelihood of two-photon correlations. When two-photon correlations are nonzero $\left(g^{(2)} \neq 0\right)$, as is often

\footnotetext{
*Corresponding author. gsolomon@umd.edu

Published by the American Physical Society under the terms of the Creative Commons Attribution 4.0 International license. Further distribution of this work must maintain attribution to the author(s) and the published article's title, journal citation, and DOI.
}

the case, it is necessary to evaluate higher-order correlations $[17,18]$. Because these correlations are derived from photon-number probability distributions, their measurement requires the accumulation of detection events. In general, to measure the photon-state statistics to $n$th order, normalized $n$ th-order correlations could be measured using a single, appropriately fast, $n$ th-order, number-resolving detector, if such a detector were available [see Fig. 1(a)] [19-21]. Alternatively, $n$ single-photon detectors can be used with beam splitters in place of an $n$ number-resolving detector [22]. For example, an $n=2$ number-resolving detector can be replaced by two single-photon detectors and a beam splitter [Fig. 1(b)], and photon detections between the two detectors can be correlated [23], as discussed in the next section.

In many quantum information applications-for instance, quantum repeater and boson sampling - the single-photon state must also be indistinguishable. The indistinguishability is measured by interfering replicas of the photon state, sampled at different times or positions [24], and can be measured with an unbalanced interferometer [see Fig. 1(c)]. However, in an unbalanced interferometer scheme, the result must account for the single-photon nature of the light [25], requiring additional information. Thus, to fully characterize the quantum state of the source-the photon-number state and the indistinguishability-at least two distinct setups and 
(a) Single spatial-

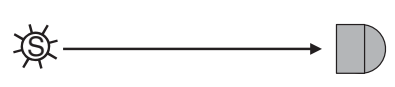

Ideal, $n$ th-order number-resolving detector

(b)

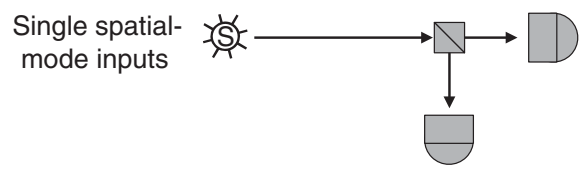

Single-photon detectors

(c)

Single spatialmode input

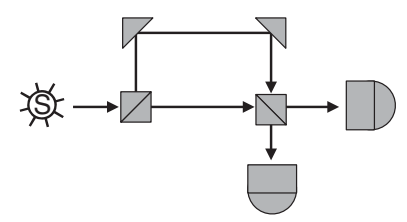

Single-photon or number-resolving detectors (a)

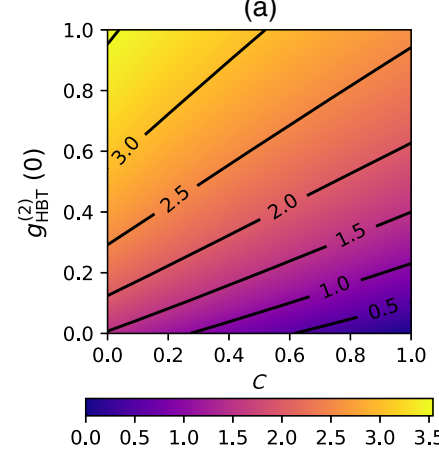

(b)

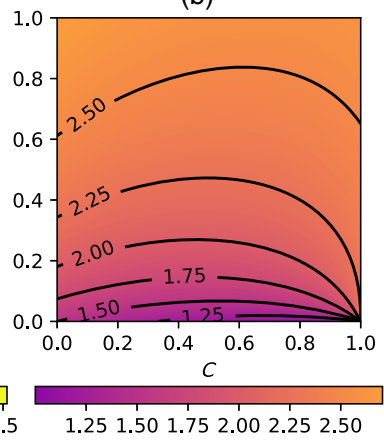

FIG. 1. Second-order characterization of light with single, spatial-mode inputs. (a) The photon-state statistics can be measured to $n$th order using a single, appropriately fast, $n$-photon number-resolving detector. Such a detector does not exist. (b) Instead of using an $n=2$ number-resolving detector, correlation measurements to second order can be made with two singlephoton (not number-resolving) detectors and a beam splitter. (c) An unbalanced interferometer can be used to interfere replicas of an input state to measure the indistinguishability from secondorder correlations. If single-photon detectors are used, an additional measurement, for instance, like the one in (b), must be made for normalization.

measurements are required. Besides the obvious inefficiencies in changing setups and acquiring separate measurement results, multiple measurements prevent the quantities defining the photon state from being evaluated from the same data.

Using two-photon $(n=2)$ number-resolving detectors in place of single-photon avalanche detectors (SPADs), we show here the simultaneous measurement of the photon state. This measurement includes the photon flux, the number-state statistics to third order, and the indistinguishability. Such a simultaneous measurement ensures that these quantities are derived from the same measurement set, and thus all aspects of the source and measurement conditions are identical. Number-resolving detectors have been demonstrated by several research groups [26,27], and they will likely be widely commercially available in the near future. We also show that the photon-number-resolved measurement intrinsically collects more information about an input photonic state than a similar measurement made with conventional detectors. Thus, regardless of setup changes, this single measurement with $n=2$ numberresolving detectors is more efficient than a combination of conventional measurements, and it becomes even more efficient as the suppression of multiphoton states (the single-photon purity) degrades. For typical assumptions of these states $\left(p_{1} \gg p_{2} \gg p_{3}\right)$, no additional advantage is gained when the photon-number resolution of the detectors is increased beyond 2. Finally, when we substitute SPADs and beam splitters for number-resolving detectors, the

FIG. 2. Comparison of the efficiency of using numberresolving detectors vs single-photon detectors with a Fisher information analysis. Values higher than 1 mean that $n=2$ number-resolving detectors are more efficient. (a) Comparison of a single measurement made with $n=2$ number-resolving detectors with the traditional (trad) two-measurement scheme, using single-photon detectors $\left(\operatorname{Tr} \mathcal{F}_{\text {trad }}^{-1} / \operatorname{Tr} \mathcal{F}_{\mathrm{n}=2}^{-1}\right)$. It can be seen that for almost all combinations of $g_{\mathrm{HBT}}^{(2)}(0)$ and $C$, it is more efficient to use number-resolving detectors. Only for very low values of $g_{\mathrm{HBT}}^{(2)}(0)$ is the traditional method more efficient. (b) Comparison of a two-measurement scheme (composite) with $n=2$ numberresolving detectors and the traditional two-measurement scheme with single-photon detectors $\left(\operatorname{Tr} \mathcal{F}_{\text {trad }}^{-1} / \operatorname{Tr} \mathcal{F}_{\text {composite } n=2}^{-1}\right)$. Here, the measurement using number-resolving detectors is always more efficient.

layout mimics a simple linear optical circuit. It can be used to model such circuits, or it can be incorporated within them for local metrology testing.

\section{SECOND-ORDER CORRELATIONS}

The use of two detectors and a beam splitter to measure the second-order normalized correlation function goes back to measurements by Hanbury Brown and Twiss (HBT) [23], and we denoted it as $g_{\mathrm{HBT}}^{(2)}$. In the HBT measurement [23], a single spatial mode is incident on one port of a beam splitter, and two detectors measure coincidences in the two output ports to assess if more than one photon is present. If $\tau$ is the difference in detection times for the two detectors, $g_{\mathrm{HBT}}^{(2)}(\tau=0)<1$ is the hallmark of a quantum state. One value of $g_{\mathrm{HBT}}^{(2)}, g_{\mathrm{HBT}}^{(2)}(0)=0$, represents a unique state. For this case, the nonvacuum component of the light is comprised of only single photons $[18,28]$. The case of $1>g_{\mathrm{HBT}}^{(2)}(0)>0$ signifies nonclassical light with some multiphoton component; the greater the value of $g_{\mathrm{HBT}}^{(2)}$, the higher the proportion of the multiphoton component in the source [29]. Thus, for a source that is expected to provide single photons, $g_{\mathrm{HBT}}^{(2)}$ is used as a metric.

A second-order correlation function can also be used to characterize the second-order interference of two input 


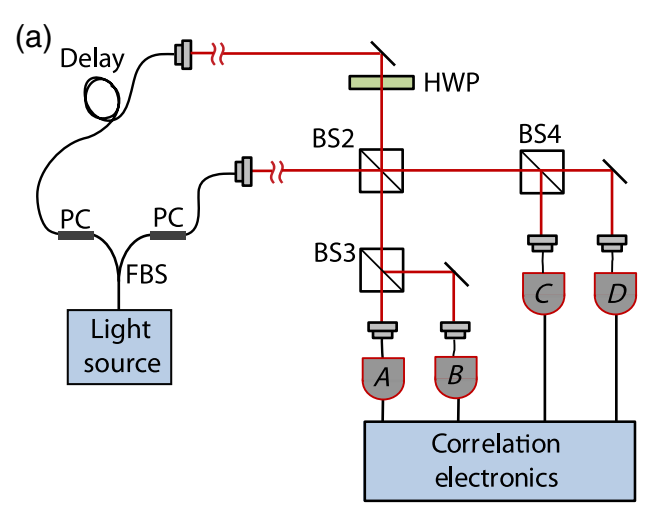

(b)

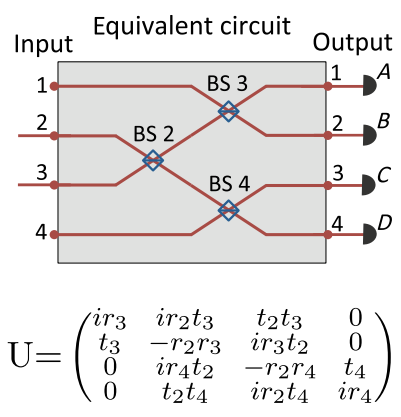

FIG. 3. (a) Measurement layout used to characterize the quantum light source. FBS is for fiber beam splitter, PC is for polarization control, HWP is the half-wave plate, BS is for beam splitter, and the detectors are A-D. The measurement arrangement consists of an unbalanced, unstabilized interferometer beginning at FBS, containing a delay line (delay) and ending at BS2. One portion is fiber (black lines), and the other portion is free space (red lines). The four detectors simulate two, two-photon, number-resolving detectors. The HWP is used in a comparison experiment (see text). (b) Equivalent optical circuit without the initial FBS and the associated unitary matrix $U$, where $r_{j}$ and $t_{j}$ are reflection and transmission coefficients for the three beam splitters.

fields, and for nonentangled inputs, it determines their indistinguishability. Using an interferometer, replicas of the same field can be used. This is often referred to as Hong, Ou, and Mandel (HOM) interferometry [24], denoted as $g_{\mathrm{HOM}}^{(2)}$. In a HOM measurement, unequal path lengths are used to match the arrival times of photons emitted at different times from a source onto a beam splitter, followed by two detectors to measure coincidences at the beam-splitter outputs. If the photons are perfectly indistinguishable single photons, both exit the same beam-splitter port [BS2 in Fig. 3(a)] making $g_{\mathrm{HOM}}^{(2)}(0)=0$ [24]. We introduce a parameter $C$, which denotes the indistinguishability independent of the singlephoton nature of the light, and we note that the measured value of $g_{\mathrm{HOM}}^{(2)}$ depends on both $C$ and $g_{\mathrm{HBT}}^{(2)}$. Thus, additional characterization is required for complete evaluation. One option is to directly determine the suppression of multiphoton states through a HBT measurement [30]. Alternatively, a second measurement [25] or series of measurements [31] can be made in which the indistinguishability is controllably varied: for example, by varying the polarization difference in each arm and thus indirectly accessing the single-photon purity [25]. The scheme presented here-using $n=2$ number-resolving detectors-provides both the multiphoton suppression and the indistinguishability with a single measurement setup.

\section{CHARACTERIZING THE EFFICIENCY}

To characterize and contrast the efficiency when using $n=2$ number-resolving detectors and single-photon detectors in evaluating single-photon-like states, we calculate the classical Fisher information $\mathcal{F}$ for each experiment. Fisher information determines how much information about a parameter can be gained from observations drawn from a given probability distribution. In our case, we have two parameters: the suppression of multiphoton states described by $g_{\mathrm{HBT}}^{(2)}(0)$ and the photon indistinguishability $C$. As discussed in more detail in the Supplemental Material, Ref. [32], the Fisher information is derived from the change in the probability distribution with the underlying parameters-in our case, $g_{\mathrm{HBT}}^{(2)}$ and $C$. Fisher information does not generally depend on the specifics of how data are evaluated, i.e., on the chosen estimator. For instance, in the traditional single-photon detector scheme, the suppression of multiphoton states is determined by a HBT measurement, while the indistinguishability is found from a HOM measurement. Nevertheless, there is some multiphoton suppression information in the HOM measurement, but it is not often used. However, a Fisher information analysis usually assumes an optimal estimator; i.e., all the information content is included, whether or not it is used. The inverse of $\mathcal{F}$ provides a lower bound on the variance of an estimator, known as the Cramér-Rao bound. We provide a detailed derivation and discussion of the Fisher information in Ref. [32].

Because we are interested in two parameters - the suppression of multiphotons and the indistinguishability-the Fisher information is contained in the $2 \times 2$ Fisher information matrix $\mathcal{F}$. After calculating the Fisher information, we compare the best achievable variance of the measurement using $n=2$ number-resolving detectors with the variance of the traditional measurement. For the traditional measurement, we use the approach by Santori et al. [30], where two sequential measurements are performed with different experimental setups: one to extract $g_{\mathrm{HBT}}^{(2)}(0)$ and one to extract $C$. Here, we calculated the Fisher information matrix for each measurement and then added the two to obtain the total Fisher information. We have chosen to set equal measurement times on both individual measurements because it cannot be known if a different distribution of measurement time would be beneficial before the 
measurement is performed. Nevertheless, one could use an adaptive measurement protocol, where more HOM or more HBT measurements are added as the measurement runs and information accumulates. For completeness, we added this comparison in Ref. [32].

In the multiparameter case, the Cramér-Rao bound is defined as $\operatorname{cov}(\theta) \geq \mathcal{F}^{-1}$, where $\operatorname{cov}(\theta)$ is the covariance matrix of the parameter estimators $\theta$. We chose to compare $\operatorname{Tr} \mathcal{F}^{-1}$ as a score in each measurement scheme. This is a comparison of the bound on the sum of the variances. Both parameters are considered equally. If one decides that $g_{\mathrm{HBT}}^{(2)}(0)$ or $C$ is more important for some reason, a different scoring could be used.

In Fig. 2(a), we plot the ratio of the scores for the traditional method (trad) and for the number-resolving detector method $(n=2), \operatorname{Tr} \mathcal{F}_{\text {trad }}^{-1} / \operatorname{Tr} \mathcal{F}_{\mathrm{n}=2}^{-1}$. Values larger than 1 mean that number-resolving detectors are more efficient, whereas values smaller than 1 mean that the single-photon detectors are more efficient. For some highindistinguishability and low- $g_{\mathrm{HBT}}^{(2)}(0)$ combinations, the traditional method is actually more efficient. For all other combinations, the number-resolving detectors are advantageous. There are two reasons that the traditional method is more efficient in certain situations. First, if the light has a low $g_{\mathrm{HBT}}^{(2)}(0)$, one does not learn more about the state by looking for two or more photons. Second, the traditional HBT measurement is very efficient in the case of low $g_{\mathrm{HBT}}^{(2)}(0)$. However, one does not know a priori the values of $g_{\mathrm{HBT}}^{(2)}(0)$ and $C$, and in general, the $n=2$ detectors provide broader improvement. This seemingly weak point of the $n=2$ number-resolving detectors can be remedied easily by complementing the single measurement with an additional HBT measurement. In Fig. 2(b), we show $\operatorname{Tr} \mathcal{F}_{\text {trad }}^{-1} / \operatorname{Tr} \mathcal{F}_{\text {composite } n=2}^{-1}$, where the $n=2$ measurement is now equally split between a HBT measurement and the full experimental apparatus outlined in this paper. It is defined in Eq. (S33) in Ref. [32]. The comparison of Figs. 2(a) and 2(b) shows that for high values of $g_{\mathrm{HBT}}^{(2)}(0)$, it is optimal to only perform a single, $n=2$ detector, HOM experiment.

We note that the plotted efficiencies are for optimal estimators. Experimentally, procedures based on optimal estimators are not usually used in the traditional method since the usual procedure is to measure $g_{\mathrm{HBT}}^{(2)}(0)$ with a HBT setup and then measure $C$ with a HOM setup. The information about $g_{\mathrm{HBT}}^{(2)}(0)$ in the HOM measurement, which is present in the central as well as the side peaks, is usually neglected (nonoptimal). We also calculated the Fisher information for the experimental case where all the side peaks in the HOM measurement are neglected for the variance of $g_{\mathrm{HBT}}^{(2)}(0)$ in keeping with common experimental realizations. Here, the advantage of $n=2$ number-resolving detectors is higher than shown in Fig. 2.
Nevertheless, we chose to plot the comparison when using optimal estimators for both measurements. This is the maximum amount of information one could get from the recorded data.

\section{EXPERIMENTAL REALIZATION}

In Fig. 3(a), a schematic of the experiment is shown. After the light source and polarization controllers, it consists of an unbalanced interferometer, followed by two beam splitters (BS3 and BS4) and four single-photon detectors. Each BS-two-detector pair combination emulates a two-photon number-resolving photon detector.

We operate under pulsed excitation and use the notation $g^{(2)}[j]$ to denote the normalized integrated correlations between two detections $j$ pulses apart. In Ref. [32], we derive functions representing $g_{\mathrm{HBT}}^{(2)}[0]$ and $C$. Note that $C$ ranges from 0 for perfectly distinguishable photons to 1 for perfectly indistinguishable photons. The results of these derivations are

$$
\begin{aligned}
g_{\mathrm{HBT}}^{(2)}[0] & =\frac{g_{\text {auto }}^{(2)}[0]+g_{\text {cross }}^{(2)}[0]-\zeta(d)}{\zeta(0)} \\
C & =\frac{g_{\text {auto }}^{(2)}[0]-g_{\text {cross }}^{(2)}[0]}{\zeta(d)},
\end{aligned}
$$

where $d$ is the delay in the unbalanced interferometer. Here, $\zeta$ accounts for temporal instabilities, in particular, the source spectral jitter, and is discussed below. Note that $g_{\text {auto }}^{(2)}[0]$ and $g_{\text {cross }}^{(2)}[0]$ are auto- and cross-correlations of the two output fields. While in Ref. [32] we account for nonideal beam splitters in the interferometer, in Eq. (1), we assume the ideal case of 50:50 beam splitters. In previous non-numberresolving two-detector schemes, just one function, $g_{\text {cross }}^{(2)}$, is measured. Since we now have two functions, both the suppression of multiphoton states and the indistinguishability can be simultaneously extracted from these quantities. Because pairs of non-number-resolving detectors are used, we simultaneously measure six of these second-order correlation functions: Two are autocorrelations of the output field $\left(g_{A B}^{(2)}\right.$ and $\left.g_{C D}^{(2)}\right)$, and four are cross-correlations $\left(g_{A C}^{(2)}\right.$, $g_{B C}^{(2)}, g_{A D}^{(2)}$, and $g_{B D}^{(2)}$ ), where $A, B, C$, and $D$ denote the four detectors in Fig. 3(a). We average them to form

$$
\begin{aligned}
g_{\text {auto }}^{(2)}[j] & =\frac{1}{2}\left(g_{A B}^{(2)}[j]+g_{C D}^{(2)}[j]\right), \\
g_{\text {cross }[j]}^{(2)}[j & =\frac{1}{4}\left(g_{A C}^{(2)}[j]+g_{A D}^{(2)}[j]+g_{\mathrm{BC}}^{(2)}[j]+g_{\mathrm{BD}}^{(2)}[j]\right) .
\end{aligned}
$$

Discrete solid-state emitters can shift in energy with time, leading to spectral jitter [33-35]. It is accounted for in Eq. (1) by the function $\zeta$, which is also discussed in Ref. [32]. If present, this jitter degrades the indistinguishably but will not degrade the suppression of multiphotons. 
Here, $\zeta$ is assumed to be a decaying exponential function of the form

$$
\zeta(k)=1+\left(\zeta_{0}-1\right) e^{-|k| / \tau_{1}},
$$

where $k$ refers to the number of pulses separating the generated photons, $\tau_{1}$ is the characteristic lifetime of the jitter (measured here in pulse periods), and $\zeta_{0}$ is the value of $\zeta$ at zero delay.

\section{MEASUREMENTS}

To demonstrate the measurement, we use photons emitted from a single InAs quantum dot (QD). Technical details about the QD device, how it is excited and how collection is made, is in Ref. [32]. It is an emerging source of bright, single-photon light [36]. A single QD typically emits light with $g_{\mathrm{HBT}}^{(2)}[0]$ close to, but different from, zero [37]. QD photon indistinguishability can vary, but normalized values above 0.95 (within 5\% of perfectly indistinguishable) have been reported [38-41].

Using the setup in Fig. 3(a), we measure the normalized second-order auto- and cross-correlations for each detector combination, as in Eq. (2). We normalize by the product of single-count probabilities $p_{l} p_{m}$, where $l$ and $m$ are the relevant detectors. The result is shown in Fig. 4.

Correlations are grouped into two categories: $g_{\text {cross }}^{(2)}[0]$ $(A C, A D, B C, B D)$ (blue, Fig. 4) and $g_{\text {auto }}^{(2)}[0](A B$ and $C D)$ (red, Fig. 4). One can qualitatively observe the nonclassical properties of the source from the matrix in Fig. 4. For instance, the larger values of the $g_{\text {auto }}^{(2)}[0]$ terms (red) compared to $g_{\text {cross }}^{(2)}[0]$ terms (blue) indicate that photons have a higher probability of leaving the same exit port of the interferometer beam splitter. This coalescence indicates photon indistinguishability.

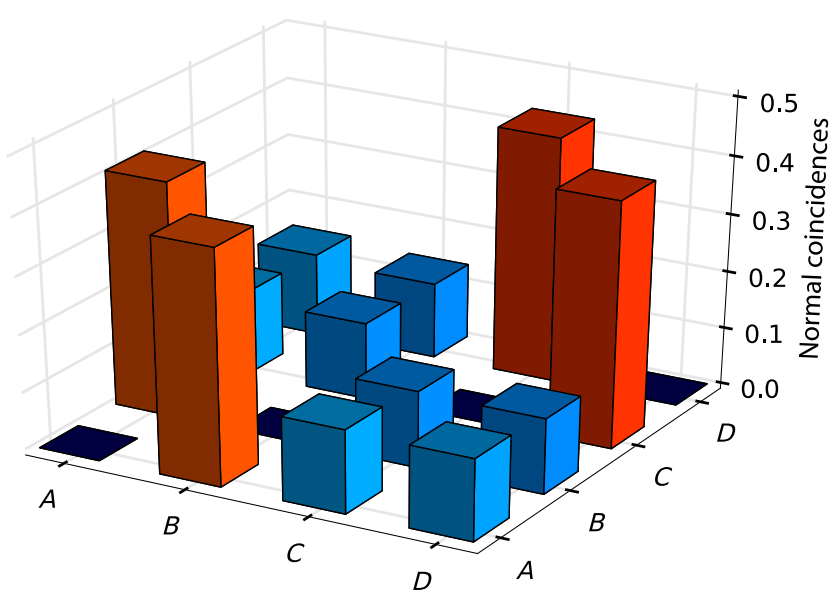

FIG. 4. Second-order characterization of quantum light-here, from a single QD source. Normalized conditional detector counts are plotted for the four detectors at $j=0$. Correlated detections on $A D, A C, B C$, and $B D$ (blue) represent cross-correlations. Correlations of the type $A B$ and $C D$ (red) are autocorrelations.
We can quantitatively extract the multi-photon suppression and the indistinguishability from the data in Fig. 4. First, using Eq. (1), we determine $g_{\mathrm{HBT}}^{(2)}[0]$. The result is shown in blue in Fig. 5. These data capture the additional dynamics associated with jitter in the QD photon frequency on a longer time scale than the QD decay. Here, $g_{\mathrm{HBT}}^{(2)}[0]=$ $0.05(1)$. Note that $\zeta_{0}=1.64$ and $\tau_{1}=3.64$ laser pulses ( $24.0 \mathrm{~ns})$. The dips at \pm 4 pulses are due to the delay in the unbalanced interferometer ( $26.3 \mathrm{~ns}=4$ laser pulses) and the single-photon nature of the source. A more detailed description of this effect is found in Ref. [32].

Determining the photon indistinguishability follows in a straightforward manner for the data in Fig. 4 using Eq. (1). We find $C=0.61(1)$. Instead of directly determining $C$, in many situations it is more convenient to associate the fringe visibility $V$ with indistinguishability, particularly when a variable controlling indistinguishably is continuously varied, for instance, the polarization [31]. For completeness, we determine it here using only one measurement set. We calculate $V$ using Eq. (S1) in Ref. [32], where it is determined directly from $g_{\mathrm{HBT}}^{(2)}[0]$ and $C$. We find $\mathrm{V}=0.58(1)$.

We compare these results with the traditional HBT measurement [23], albeit with four detectors instead of the usual two, and the indistinguishability results with the traditional measurement made in two steps. The traditional HBT measurement is done without the first beam splitter, i.e., bypassing the interferometer and summing pair correlations over the four detectors. The comparison is shown in green in Fig. 5. The measurements compare well. Here, $g_{\mathrm{HBT}}^{(2)}[0]=0.060(6)$ using the standard HBT configuration.

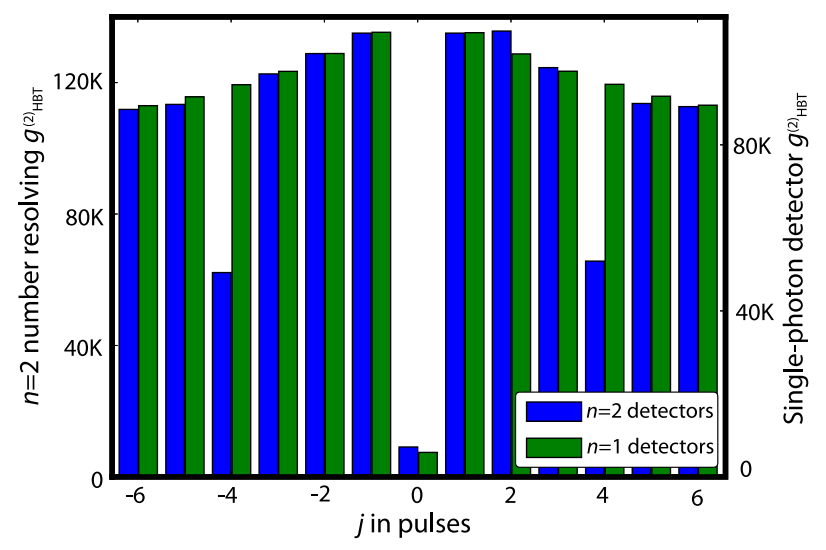

FIG. 5. A comparison of the $g_{\mathrm{HBT}}^{(2)}$ measurements, uncorrected for spectral jitter. The $g_{\mathrm{HBT}}^{(2)}$ data for the number-resolving detector measurement (blue) are extracted from the data in Fig. 4 and compare well to the traditional HBT measurement (green). The two measurements are off-set laterally for clarity. The dip at \pm 4 pulses is due to the interferometer (and single-photon character of the source) (see text and Ref. [32] for details). Using Eq. (1), $g_{\mathrm{HBT}}^{(2)}[0]$ can be found. 
Using the $g_{\mathrm{HBT}}^{(2)}$ data and Eq. (3), we obtain the same values for $\zeta_{0}$ and $\tau_{1}$. The dip at four laser pulses is not present here because the unbalanced interferometer is not used.

To determine the indistinguishability in the traditional way, the value of $g_{\mathrm{HBT}}^{(2)}[0]$ needs to be known, and a second measurement with the interferometer determines $g_{\mathrm{HOM}}^{(2)}$. Here, the indistinguishability is found from Ref. [25], where the probability of coalescence is $C=$ $1+g_{\mathrm{HBT}}^{(2)}[0]-2 g_{\mathrm{HOM}}^{(2)}[0]$. The coalescence is $0.62(1)$, in good agreement with the new technique utilizing a single measurement. We also determine $V$ using the traditional method. It requires two measurements, where the half-wave plate (HWP) in Fig. 3(a) is set to rotate the polarization in one arm of the interferometer by 0 or by $\pi / 2$. Using the traditional approach, we find $V=0.58(9)$, equal to, within error, the number-resolving detector result. The error is larger here because of the small data set for $g_{\mathrm{HOM}}^{(2)}$ when the HWP makes the two paths distinguishable.

\section{MEASURING THE PROBABILITY DISTRIBUTION}

To fully characterize the photon-number distribution, higher-order correlation measurements are generally required $[18,42]$. Characterization to at least third order is necessary. Using the standard HBT-type measurement with two single-photon detectors, second-order correlations determine photon-number statistics to only second order $(n=2)$ [43]. Using the $n=2$ number-resolving detectors, we can determine a probability distribution of the photonnumber states up to $n=3$. For the QD light source used here, a photon should be emitted from the source every laser-pump cycle $(2 \times 76 \mathrm{MHz})$; however, the system is only pumped to $70 \%$ of saturation. We determine the photon count rate at the fiber exiting our cryostat (just before the interferometer input) to be $3.08 \times 10^{6} \mathrm{cts} / \mathrm{s}$, indicating the source efficiency $\left(p_{1}\right)$ exiting the fiber is 0.020 . Here, $p_{1}$ is calculated based on the detector efficiencies and transmission through the interferometer, and these were measured to be $0.65 \%$. Then $p_{0} \approx$ $1-p_{1}$ is directly calculated to be 0.98 and $p_{2}$ is determined from $g_{H B T}^{(2)}[0]=2 p_{2} / p_{1}^{2}$ to be $1.0 \times 10^{-5}$. The uncertainties on the above values are dominated by the long-term fluctuations in the setup, which we estimate to be $10 \%$. Zero three- or four-photon coincidences were measured for a trial number of $1.82 \times 10^{13}$, giving an upper limit of $p_{3}$ of $2.1 \times 10^{-6}$. The assumption of $p_{0} \gg p_{1} \gg p_{2} \gg p_{N>2}$ holds, and the QD emission is described by a mixed state with a density matrix $\|p\|=0.98 p_{0,0}+0.020 p_{1,1}+\left(1.0 \times 10^{-5}\right) p_{2,2}+\sum_{i>2} 0 p_{i, i} \times$ $\left(+2.1 \times 10^{-6}\right)$, where $p_{n, m}=\left|f_{n}\right\rangle\left\langle f_{m}\right|$, and $n, m$ are elements of the density matrix. We note that the off-diagonal elements of $p$ are expected to be zero; i.e., there is no coherence between different number states.

\section{CONCLUSIONS}

Using the light emission from a semiconductor QD structure as a test light source, we have demonstrated a new measurement approach, allowing simultaneous measurement of the brightness, suppression of multiphotons, and photon indistinguishability. The approach uses an interferometer and two two-photon number-resolving detectors, here simulated by four detectors. The simultaneous second-order correlation measurement proposed here eliminates any variation in source and experiment that may be present in independent measurements, and in nearly all cases, it does so with reduced uncertainty. The measurement is especially efficient when the nonclassical light has less than ideal single-photon properties. Finally, while $n=2$ number-resolving detectors are the critical elements in this approach, higher-order number-resolving detectors do not offer improved efficiency, although they would be useful to characterize higher-order number states.

As an additional remark, the number-resolving detector measurement can be evaluated using a photonic circuit approach, as seen in the equivalent circuit in Fig. 3(b). The unitary matrix of this circuit, Fig. 3(c), can be used to determine the $g_{\mathrm{HBT}}^{(2)}[0]$ and $C$ values that most closely match the Fock-state distribution [8] found for the QD source. The $g_{\mathrm{HBT}}^{(2)}[0]$ and $C$ determined by the model that best matches Fig. 4 are within the uncertainty of those previously determined. This measurement could be incorporated into complex photonic circuits to assess the second-order correlation properties of the light.

Number-resolving detectors, as well as QD-based singlephoton sources like the one used here, are emerging technologies that will likely have a strong impact in quantum measurement, experiments, and systems. While we have shown that $n=2$ number-resolving detectors will advance the characterization of quantum light, we believe they will also improve a diverse set of quantum optics experiments, for instance, the boson sampling class of problems discussed above. We hope this work helps to further motivate such efforts.

\section{ACKNOWLEDGMENTS}

This work was partially supported by the NSF PFC@ JQI and the Army Research Laboratory. We thank P. Senellart and A. Gorshkov for helpful discussions and a critical reading of the manuscript. S. V.P. thanks Pavel E. Samoylov for encouraging discussions. Z. E. is supported in part by the ARCS Foundation, ARL CDQI, ARO MURI, NSF QIS, ARO, NSF PFC at JQI, and AFOSR. G. S. S. acknowledges support from Fulbright Austria-Austrian American Educational Commission through the FulbrightUniversity of Innsbruck Visiting Scholar program. 
[1] D. E. Browne and T. Rudolph, Resource-Efficient Linear Optical Quantum Computation, Phys. Rev. Lett. 95, 010501 (2005).

[2] M. Zwerger, W. Dür, and H. J. Briegel, Measurement-Based Quantum Repeaters, Phys. Rev. A 85, 062326 (2012).

[3] N. Sangouard, C. Simon, H. de Riedmatten, and N. Gisin, Quantum Repeaters Based on Atomic Ensembles and Linear Optics, Rev. Mod. Phys. 83, 33 (2011).

[4] S. Muralidharan, L. Li, J. Kim, N. Lütkenhaus, M. D. Lukin, and L. Jiang, Efficient Long Distance Quantum Communication, Sci. Rep. 6, 20463 (2016).

[5] A. Aspuru-Guzik and P. Walther, Photonic Quantum Simulators, Nat. Phys. 8, 285 (2012).

[6] M. A. Broome, A. Fedrizzi, J. Dove, S. Aaronson, T. C. Ralph, and A. G. White, Photonic Boson Sampling in a Tunable Circuit, Science 339, 794 (2013).

[7] J. B. Spring, B. J. Metcalf, P. C. Humphreys, W. S. Kolthammer, X.-M. Jin, M. Barbieri, A. Datta, N. Thomas-Peter, N. K. Langford, D. Kundys, J. C. Gates, B. J. Smith, P. G. R. Smith, and I. A. Walmsley, Boson Sampling on a Photonic Chip, Science 339, 798 (2013).

[8] M. Tillmann, B. Dakić, R. Heilmann, S. Nolte, A. Szameit, and P. Walther, Experimental Boson Sampling, Nat. Photonics 7, 540 (2013).

[9] A. Crespi, R. Osellame, R. Ramponi, D. J. Brod, E. F. Galvão, N. Spagnolo, C. Vitelli, E. Maiorino, P. Mataloni, and F. Sciarrino, Integrated Multimode Interferometers with Arbitrary Designs for Photonic Boson Sampling, Nat. Photonics 7, 545 (2013).

[10] J. Carolan, C. Harrold, C. Sparrow, E. Martín-López, N. J. Russell, J. W. Silverstone, P. J. Shadbolt, N. Matsuda, M. Oguma, M. Itoh et al., Universal Linear Optics, Science 349, 711 (2015).

[11] J. D. Franson, Nonlocal Cancellation of Dispersion, Phys. Rev. A 45, 3126 (1992).

[12] A. M. Steinberg, P. G. Kwiat, and R. Y. Chiao, Dispersion Cancellation in a Measurement of the Single-Photon Propagation Velocity in Glass, Phys. Rev. Lett. 68, 2421 (1992).

[13] C. M. Caves, K. S. Thorne, R. W. P. Drever, V. D. Sandberg, and M. Zimmermann, On the Measurement of a Weak Classical Force Coupled to a Quantum-Mechanical Oscillator. I. Issues of Principle, Rev. Mod. Phys. 52, 341 (1980).

[14] S. Dolinar, Jr., Research Laboratory of Electronics Report No. RLE_QPR_111_VII, 1973, http://hdl.handle.net/1721 $.1 / 56414$.

[15] R. L. Cook, P. J. Martin, and J. M. Geremia, Optical Coherent State Discrimination Using a Closed-Loop Quantum Measurement, Nature (London) 446, 774 (2007).

[16] R. J. Glauber, Coherent and Incoherent States of the Radiation Field, Phys. Rev. 130, 2529 (1963). Here the unnormalized $g^{(1)}$ is denoted $G^{(1)}$.

[17] L. Zhang, H. B. Coldenstrodt-Ronge, A. Datta, G. Puentes, J. S. Lundeen, X.-M. Jin, B. J. Smith, M. B. Plenio, and I. A. Walmsley, Mapping Coherence in Measurement via Full Quantum Tomography of a Hybrid Optical Detector, Nat. Photonics 6, 364 (2012).

[18] E. Goldschmidt, F. Piacentini, I. R. Berchera, S. V. Polyakov, S. Peters, S. Kück, G. Brida, I. P. Degiovanni, A. Migdall, and M. Genovese, Mode Reconstruction of a
Light Field by Multiphoton Statistics, Phys. Rev. A 88, 013822 (2013).

[19] For example, transition-edge sensors are currently available and have shown unsaturated photon-number resolution for low photon-number states (up to $\approx 10$ photons), but they currently may lack temporal resolution for rapid sources such as the source discussed here.

[20] A. E. Lita, A. J. Miller, and S. W. Nam, Counting NearInfrared Single-Photons with 95\% Efficiency, Opt. Express 16, 3032 (2008).

[21] Z. H. Levine, T. Gerrits, A. L. Migdall, D. V. Samarov, B. Calkins, A. E. Lita, and S. W. Nam, Algorithm for Finding Clusters with a Known Distribution and Its Application to Photon-Number Resolution Using a Superconducting Transition-Edge Sensor, J. Opt. Soc. Am. B 29, 2066 (2012).

[22] M. J. Stevens, S. Glancy, S. W. Nam, and R. P. Mirin, ThirdOrder Antibunching from an Imperfect Single-Photon Source, Opt. Express 22, 3244 (2014).

[23] R. Hanbury Brown and R. Q. Twiss, Correlation Between Photons in Two Coherent Beams of Light, Nature (London) 177, 27 (1956).

[24] C. K. Hong, Z. Y. Ou, and L. Mandel, Measurement of Subpicosecond Time Intervals Between Two Photons by Interference, Phys. Rev. Lett. 59, 2044 (1987).

[25] E. B. Flagg, S. V. Polyakov, T. Thomay, and G. S. Solomon, Dynamics of Nonclassical Light from a Single Solid-State Quantum Emitter, Phys. Rev. Lett. 109, 163601 (2012).

[26] D. Rosenberg, A. Lita, A. Miller, and S. Nam, Noise-Free High-Efficiency Photon-Number-Resolving Detectors, Phys. Rev. A 71, 061803 (2005).

[27] A. Divochiy, F. Marsili, D. Bitauld, A. Gaggero, R. Leoni, F. Mattioli, A. Korneev, V. Seleznev, N. Kaurova, O. Minaeva et al., Superconducting Nanowire Photon-Number-Resolving Detector at Telecommunication Wavelengths, Nat. Photonics 2, 302 (2008).

[28] L. Mandel and E. Wolf, Optical Coherence and Quantum Optics (Cambridge University Press, Cambridge, England, 1995).

[29] H. J. Kimble, M. Dagenais, and L. Mandel, Photon Antibunching in Resonance Fluorescence, Phys. Rev. Lett. 39, 691 (1977).

[30] C. Santori, D. Fattal, J. Vucković, G. S. Solomon, and Y. Yamamoto, Indistinguishable Photons from a SinglePhoton Device, Nature (London) 419, 594 (2002).

[31] P. G. Kwiat, A. M. Steinberg, and R. Y. Chiao, Observations of a "Quantum Eraser": A Revival of Coherence in a Two-Photon Interference Experiment, Phys. Rev. A 45, 7729 (1992).

[32] See Supplemental Material at http://link.aps.org/ supplemental/10.1103/PhysRevX.7.041036 for more details and discussions related to this letter.

[33] A. V. Kuhlmann, J. H. Prechtel, J. Houel, A. Ludwig, D. Reuter, A. D. Wieck, and R. J. Warburton, TransformLimited Single Photons from a Single Quantum Dot, Nat. Commun. 6, 8204 (2015).

[34] A. Thoma, P. Schnauber, M. Gschrey, M. Seifried, J. Wolters, J.-H. Schulze, A. Strittmatter, S. Rodt, A. Carmele, A. Knorr, T. Heindel, and S. Reitzenstein, Exploring Dephasing of a Solid-State Quantum Emitter via Time- 
and Temperature-Dependent Hong-Ou-Mandel Experiments, Phys. Rev. Lett. 116, 033601 (2016).

[35] J. C. Loredo, N. A. Zakaria, N. Somaschi, C. Anton, L. de Santis, V. Giesz, T. Grange, M. A. Broome, O. Gazzano, G. Coppola et al., Scalable Performance in Solid-State Single-Photon Sources, Optica 3, 433 (2016).

[36] O. Gazzano and G. S. Solomon, Toward Optical Quantum Information Processing with Quantum Dots Coupled to Microstructures, J. Opt. Soc. Am. B 33, C160 (2016).

[37] P. Michler, A. Imamoglu, M. D. Mason, P. J. Carson, G. F. Strouse, and S. K. Buratto, Quantum Correlation Among Photons from a Single Quantum Dot at Room Temperature, Nature (London) 406, 968 (2000).

[38] O. Gazzano, S. Michaelis de Vasconcellos, C. Arnold, A. Nowak, E. Galopin, I. Sagnes, L. Lanco, A. Lemaître, and P. Senellart, Bright Solid-State Sources of Indistinguishable Single Photons, Nat. Commun. 4, 1425 (2013).

[39] Y.-M. He, Y. He, Y.-J. Wei, D. Wu, M. Atatü, C. Schneider, S. Höping, M. Kamp, C.-Y. Lu, and J.-W. Pan, On-Demand Semiconductor Single-Photon Source with Near-Unity Indistinguishability, Nat. Nanotechnol. 8, 213 (2013).
[40] Y.-j. Wei, Y.-M. He, M.-c. Chen, Y.-n. Hu, Y. He, D. Wu, C. Schneider, M. Kamp, S. Höfling, C.-Y. Lu, and J.-W. Pan, Deterministic and Robust Generation of Single Photons on a Chip with 99.5\% Indistinguishability Using Rapid Adiabatic Passage, Nano Lett. 14, 6515 (2014).

[41] A. K. Nowak, S. L. Portalupi, V. Giesz, O. Gazzano, C. Dal Savio, P.-F. Braun, K. Karrai, C. Arnold, L. Lanco, I. Sagnes, A. Lemaître, and P. Senellart, Deterministic and Electrically Tunable Bright Single-Photon Source, Nat. Commun. 5, 3240 (2014).

[42] M. Stevens, Photon statistics, measurements, and measurements tools, in Single-Photon Generation and Detection, Vol. 45: Physics and Applications (Experimental Methods in the Physical Sciences), edited by A. Migdall, S. V. Polyakov, J. Fan, and J. C. Bienfang, 1st ed. (Academic Press, New York, 2013), Chap. 2, pp. 25-66.

[43] A. Predojević, M. Ježek, T. Huber, H. Jayakumar, T. Kauten, G. S. Solomon, R. Filip, and G. Weihs, Efficiency vs. MultiPhoton Contribution Test for Quantum Dots, Opt. Express 22, 4789 (2014). 